Review

\title{
Spotlight on Aspergillus nidulans photosensory systems
}

\author{
Özgür Bayram ${ }^{a}$, Gerhard H. Braus ${ }^{\mathrm{a}, *}$, Reinhard Fischer ${ }^{\mathrm{b}, * *}$, Julio Rodriguez-Romero ${ }^{\mathrm{b}}$ \\ ${ }^{a}$ Institute of Microbiology and Genetics, Dept. of Molecular Microbiology and Genetics, Georg August University of Göttingen, Grisebachstr. 8, D-37077 Göttingen, Germany \\ ${ }^{\mathrm{b}}$ Karlsruhe Institute of Technology (KIT) - South Campus, Institute for Applied Biosciences, Dept. of Microbiology, Hertzstr. 16, D-76187 Karlsruhe, Germany
}

\section{A R T I C L E I N F O}

\section{Article history:}

Received 12 February 2010

Accepted 19 May 2010

Available online 23 May 2010

\section{Keywords:}

Aspergillus

Red-light

Blue-light

Phytochrome

LreA

LreB

CryA

VeA

Velvet complex

\begin{abstract}
A B S T R A C T
Aspergilli are ubiquitous soil-borne fungi growing within or on the surface of numerous organic substrates. Growth within a substrate or growth on the surface correlates to different growth conditions for the hyphae due to significant changes in oxygen or reactive oxygen species levels and variations in humidity or temperature. The production of air-borne spores is supported by the substrate-air interphase and also requires a sensing system to adapt appropriately. Here we focus on light as important parameter for the mycelium to discriminate between different habitats. The fungal 'eye' includes several light sensors which react to a broad plethora of wavelengths. Aspergillus nidulans light receptors comprise a phytochrome for red-light sensing, white collar-like blue-light signaling proteins, a putative green-light sensing opsin and a cryptochrome/photolyase as distinct sensory systems. Red- and blue-light receptors are assembled into a light-sensing protein complex. Light receptors transmit their signal to a number of other regulatory proteins including a bridging protein, VeA, as part of a trimeric complex. VeA plays a central role in the balance of asexual and sexual development and in the coordination of morphogenesis and secondary metabolism.
\end{abstract}

(ㄷ) 2010 Elsevier Inc. All rights reserved.

\section{Contents}

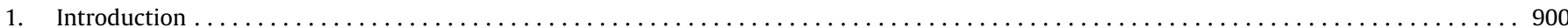

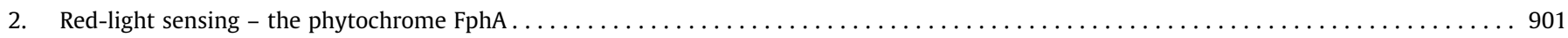

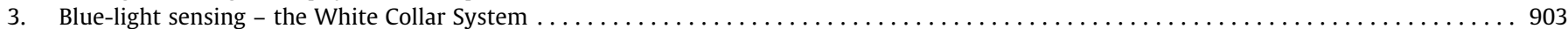

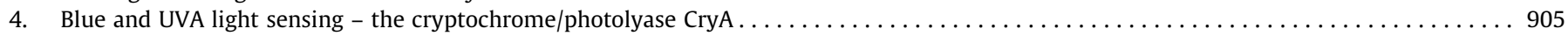

5. The velvet complex: branchpoint of light signaling in coordinated control of development and secondary metabolism .......... 905

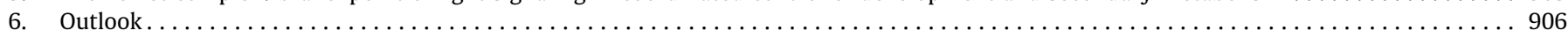

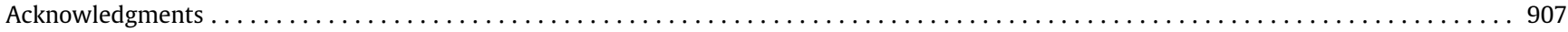

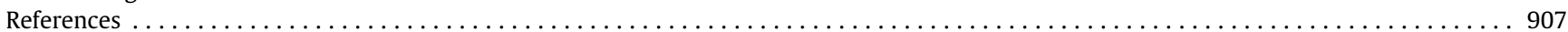

\section{Introduction}

The filamentous fungus Aspergillus nidulans represents the scientific model organism for a large group of over 185 Aspergilli (Samson, 1992) including several species with beneficial or deleterious impacts on human health. Aspergillus oryzae and Aspergillus niger are of high importance to produce sake, miso, soy sauce, and citric acid in food industry. Aspergilli can produce bioactive molecules like penicillin, and Aspergillus fumigatus and Aspergillus flavus, which can cause invasive aspergillosis in immunocompro-

\footnotetext{
* Corresponding author. Fax: +49 0551393330.

** Corresponding author. Fax: +49 07216084509.

E-mail addresses: gbraus@gwdg.de (G.H. Braus), reinhard.fischer@kit.edu (R. Fischer).
}

mised patients, secrete also gliotoxin or aflatoxins (Brakhage et al., 2004; Gugnani, 2003; Hedayati et al., 2007). A. nidulans whose genetics is well established produces the aflatoxin precursor sterigmatocystin. The $A$. nidulans genome is available and can be compared to various other genomes of Aspergilli (Draht et al., 2007; Galagan et al., 2005; Yu and Keller, 2005).

A. nidulans has been used as a model to study the cell biology and morphogenesis of filamentous fungi (Adams et al., 1998; Fischer and Kües, 2006). Especially the asexual developmental pathway has been studied in great detail. After germination of the spores, a phase of about $20 \mathrm{~h}$ is required for the hyphae to acquire developmental competence. If then the environmental conditions are in favor of development (such as air exposure and light), some hyphal compartments differentiate into a thick-walled foot, from which a ca. $70 \mu \mathrm{m}$ long stalk emerges (Fig. 1A). This swells terminally to 
A

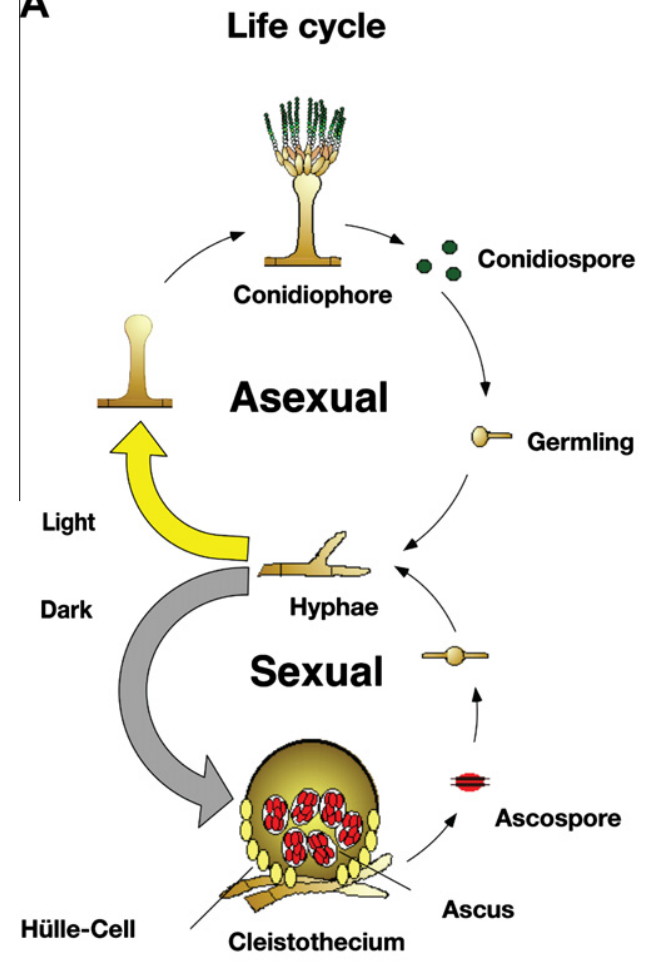

B

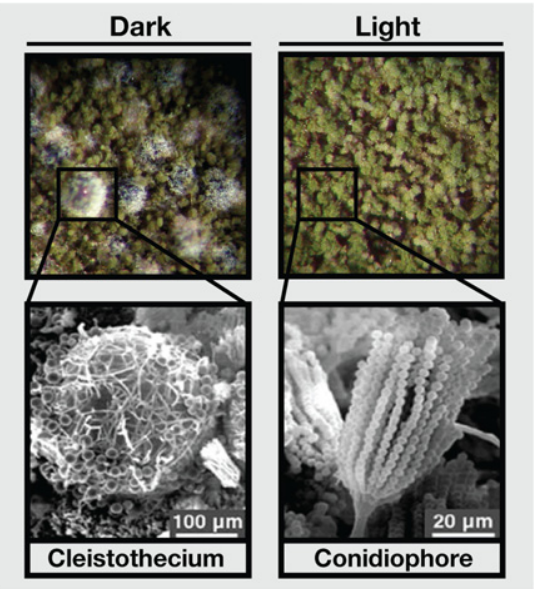

C

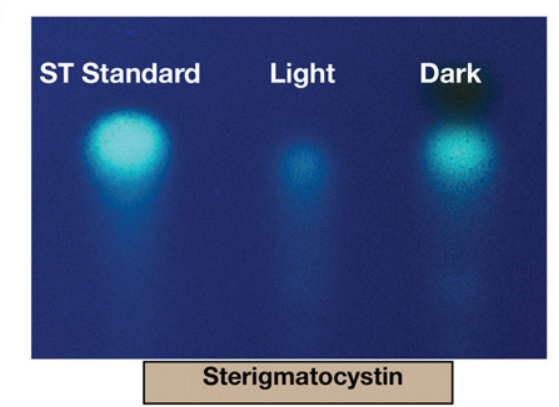

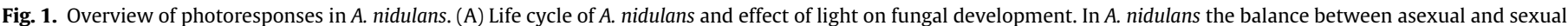

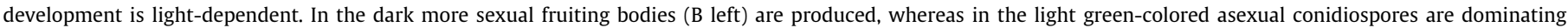

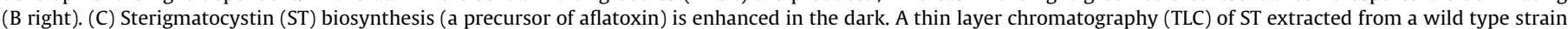

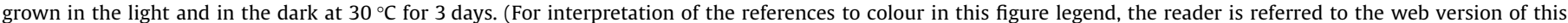
article.)

form the vesicle from which 50-70 metulae are produced, which itself produce 2-3 phialides each. Whereas metulae contain a single nucleus, which does not divide anymore, phialides are mitotically highly active and generate long chains of single nuclear conidiospores. The genetics underlying this fascinating process has been revealed through the analysis of developmental mutants (Clutterbuck, 1969; Timberlake, 1987). A class of early genes has been defined and named fluffy due to the cotton-like appearance of the mycelium of corresponding mutants (Lee and Adams, 1994). Whereas the exact signaling processes involving the fluffy genes, is not completely solved, a cascade of transcription factors leads to the activation of the bristle A master regulator gene (Adams et al., 1990). This in turn controls a gene for a second transcription factor, abacus $A$, and together they control the gene activity of many developmental genes (Andrianopoulos and Timberlake, 1994). If the conditions are no longer favorable for vegetative growth, $A$. nidulans is able to undergo the sexual cycle (Fig. 1A). It first produces small nests where first primordia of cleistothecia are formed. Growing primordia undergo drastic differentiation finally resulting in approximately $200 \mathrm{~mm}$ size spherical closed fruiting bodies called cleistothecia. Cleistothecia are surrounded by the globose Hülle cells whose function although debatable is to provide nutrients for the developing cleistothecia. Cleistothecia contain meiotically produced binuclear ascospores (Braus et al., 2002; Busch and Braus, 2007) (Fig. 1A). During the last decade a couple of genes including $n s d D$, nos $A$, veA, $r c o A$ necessary for cleistothecia formation have been studied (Han et al., 2001; Kim et al., 2002; Todd et al., 2006; Vienken and Fischer, 2006).

Recently, it has been recognized that the control of the developmental programs (asexual and sexual development) are coordinated with the control of secondary metabolism (Fig. 1C). Likewise, mutations resulting in defects in fungal development often also impair secondary metabolism (Calvo et al., 2002). Light represents one of several environmental cues which trigger and coordinate these different processes (Braus et al., 2002; Busch and Braus, 2007; Pöggeler et al., 2006). Fungal light sensors and subsequent light control is a major focus of fungal research of the past years. Most of the work had originally been performed with the Neurospora crassa light control. Intensive work on light sensing in the model system $A$. nidulans is more recent and the focus is on light dependent development. $A$. nidulans forms asexual spores in light, but preferentially undergoes sexual reproduction in the dark (Adams et al., 1998; Mooney and Yager, 1990; Purschwitz et al., 2006). Light controlled-development of $A$. nidulans is provided by several sensors: FphA is a phytochrome representing a red-light receptor which represses fruitbody formation and induces asexual spore formation (Blumenstein et al., 2005). The white-collar complex (WCC) LreA (WC-1) and LreB (WC-2) (Purschwitz et al., 2008) and the photolyase/cryptochrome CryA (Bayram et al., 2008a; Purschwitz et al., 2008) represent blue-light receptors. Knowledge about a gene for an opsin within the $A$. nidulans genome is scarce and the function of the protein is not yet explored in detail. Another light-dependent regulator protein affecting secondary metabolism and development is the velvet factor VeA which is present in many ascomycetes (Calvo, 2008; Kaefer, 1965; Kato et al., 2003) and which represents a light sensitive bridging protein in one or two protein complexes which coordinate development and secondary metabolism (Bayram et al., 2008b). This review summarizes the major findings for these Aspergilli light sensors.

\section{Red-light sensing - the phytochrome FphA}

Already more than 40 years ago red-light responses have been described in fungi. In Botrytis cinerea and Alternaria solani sporula- 
tion is inhibited by blue light but the effect could be reverted by red light illumination (Lukens, 1965; Tan, 1974). Another prominent example is $A$. nidulans. Whereas under light conditions, asexual conidiospores are formed, the fungus produces resistant and durable ascospores in the dark (Fig. 1A). However, the photobiology has been largely neglected, because early on a 'blind' mutant strain, named veA1, had been isolated, which produced large amounts of asexual spores also in the absence of light. This was convenient for the cultivation of the fungus in laboratories and was not likely to interfere in experiments studying the metabolism. However, even the molecular analysis of a bona-fide lightdependent process, the asexual sporulation, was mainly performed in a veA1 mutant background. Despite the obvious importance of the $v e A$ gene, the molecular analysis of this gene was only performed quite recently and revealed that it encodes a regulator but not a light sensor (see below) (Kim et al., 2002). Only in a few publications, the light response of $A$. nidulans has been studied in some detail. Photobiological experiments suggested already 1990 that a phytochrome might be involved in the regulation (Mooney and Yager, 1990). However, the dogma at that time was, that phytochromes are plant-specific molecules, and it took until 2005 that first fungal phytochromes were functionally characterized. Some years before, phytochrome has already been identified in the cyanobacteria Synechocystis PCC6803 and Fremyella diplosiphon (Kaneko et al., 1996; Kehoe and Grossman, 1996; Lamparter et al., 1997) and even more surprisingly, in the heterotrophic bacteria Deinococcus radiodurans and Pseudomonas aeruginosa (Davis et al., 1999). These discoveries suggested that phytochrome must have evolved millions of years before the emergence of green plants.

Phytochromes are light-sensor proteins, associated with a linear tetrapyrrol (bilin) as the chromophore. Plant phytochromes are synthesized in the cytoplasm as $\approx 120 \mathrm{kDa}$ monomers (Quail, 2002; Rockwell et al., 2006) and the chromophore attaches autocatalytically to a conserved cysteine. Phytochrome exists in two convertible forms that are distinguished by their absorption maxima and structure. They are synthesized in the red light absorbing
Pr conformation. Absorption of red light first leads to cis-trans isomerization of the chromophore followed by changes of the protein conformation. The ratio between the two states determines the signaling state of the phytochrome. Phytochromes are proteins with a modular architecture consisting of an N-terminal photosensory module (GAF and PHY domain) harboring the open-chain tetrapyrrol chromophore (Fig. 3). Whereas plant and cyanobacterial phytochromes incorporate phytochromobilin (РФВ) and phycocyanobilin (PCB) respectively, BphPs and Fphs bind the more oxidized biliverdin IX $\alpha(B V)$. The C-terminal output domain varies strongly amongst different species but always contains a histidine kinase related domain (HKRD) that is involved in the regulatory output.

The supply of the bilin chromophore is achieved in several steps in plants. The oxidative cleavage of the precursor molecule heme is performed by a heme oxygenase and leads to the production of $\mathrm{BV}$. For the synthesis of PФB and PCB an additional reduction step catalyzed by a bilin reductase is needed. Since no heme oxygenase can be identified in most of the as yet available fungal genomes, the nature and synthesis of the bilin chromophore remains to be elucidated. In Saccharomyces cerevisiae and Candida albicans heme oxygenases exist and are used to acquire iron from heme (Kim et al., 2006), but these fungi do not possess phytochrome. It has been shown that $A$. nidulans FphA, expressed in Escherichia coli, assembles efficiently with biliverdin. However, whether this is also the natural chromophore has not been shown yet (Blumenstein et al., 2005).

The function of phytochrome as light sensor has been shown in A. nidulans. Deletion of the gene in $A$. nidulans caused a slight reduction of the light effect (Fig. 2B), suggesting the involvement of this photoreceptor in the light response. The fact that the fphA mutant was still able to respond to light pointed to the presence of other photoreceptors (see below).

All the observed light-dependent changes are most likely brought about by differential gene expression. Indeed, genome wide analyses in N. crassa and Trichoderma atroviride revealed that about $3 \%$ of all genes are light regulated (Lewis et al., 2002;
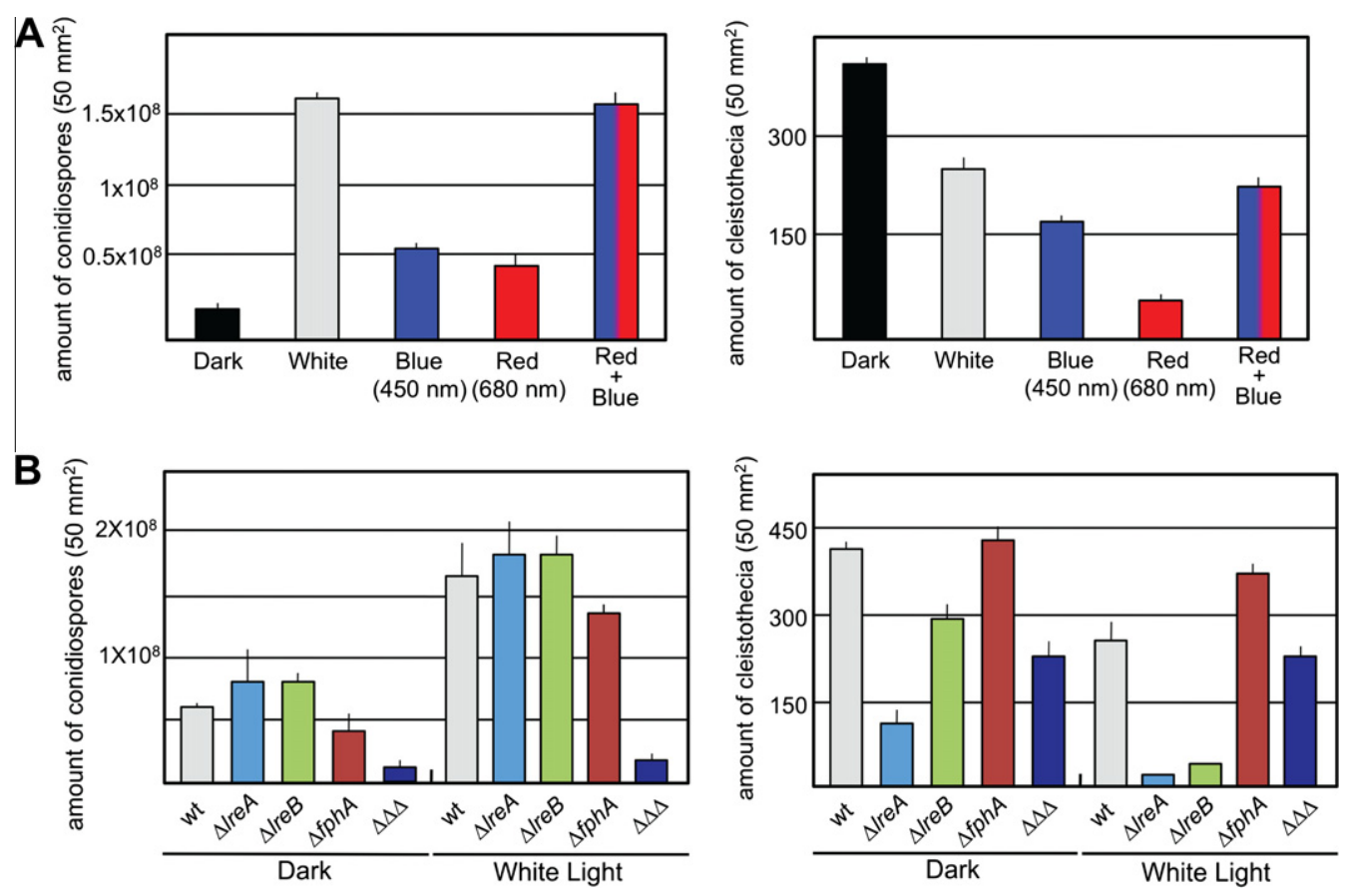

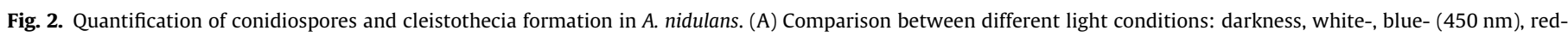

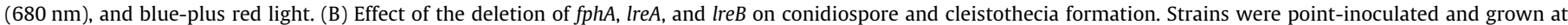

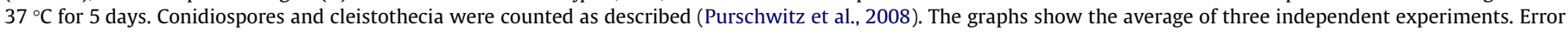
bars represent the standard deviations. (For interpretation of the references to colour in this figure legend, the reader is referred to the web version of this article.) 


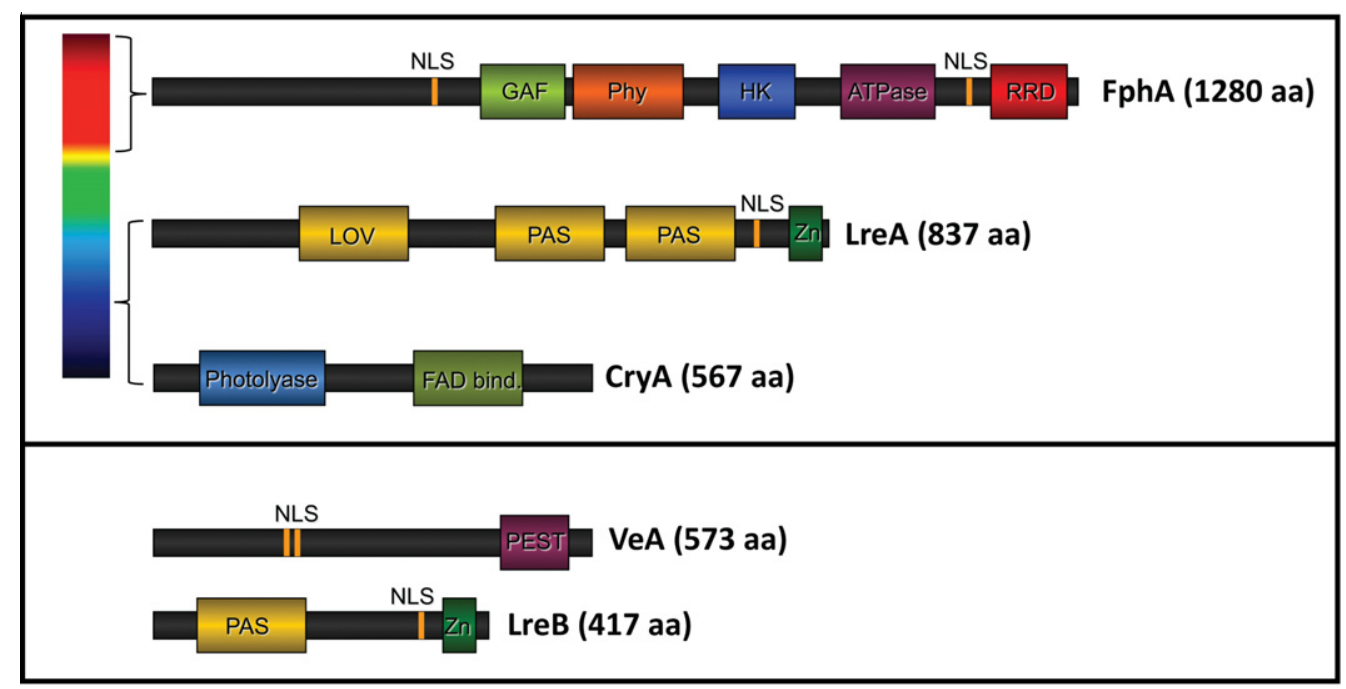

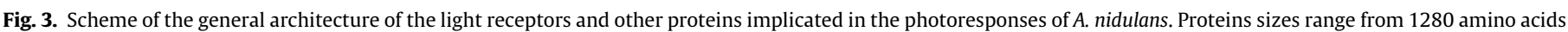
for the A. nidulans FphA protein to 417 amino acids for the LreB protein. The nomenclature of the domains is explained in the text.

Rosales-Saavedra et al., 2006). This observation leads to the next interesting question regarding signal transduction after light absorption. In plants it has been shown that phytochrome shuttles from the cytoplasm to the nucleus upon illumination (Sakamoto and Nagatani, 1996). In the nucleus it interacts with, e.g. transcription factors which in turn regulate the transcription of several genes. In $A$. nidulans phytochrome was found in the cytoplasm and in the nucleus, where it forms a light-sensing protein complex (see below). However, there is no evidence for a shuttling mechanism yet. A second possibility for light-dependent activity changes, is the phosphorylation status of the protein and a putative kinase activity of the output module. Fungal phytochromes are more closely related to bacterial than to plant phytochromes, which is highlighted by the fact that a complete output module consisting of a HKD and RRD is encoded within the same gene/ORF as the chromophore binding domain (Karniol et al., 2005). The N. crassa genome encodes even two phytochromes, PHY-1/2, both of which contain RR motifs at their C-terminus (Froehlich et al., 2005). But so far only for the A. nidulans phytochrome FphA a light driven histidine kinase activity could be determined (Brandt et al., 2008). It has been shown that FphA forms dimers and that a conserved cysteine residue 195 within the PAS domain serves as the chromophore attachment site. After binding to biliverdin, FphA shows spectral properties of a typical phytochrome (Blumenstein et al., 2005). The form initially synthesized in the dark is the Pr form (max. $707 \mathrm{~nm}$ ) that turns into Pfr (max. $754 \mathrm{~nm}$ ) under red light illumination. Dark reversion, the thermal relaxation observed in plant phytochromes, has not been observed. However, dark reversion could be released after deletion of the $\mathrm{N}$-terminal variable extension (NTE). In this context it was shown that the NTE comprising the first 172 amino acids is sufficient to stabilize the Pfr form of FphA (Brandt et al., 2008). In protein kinase assays a light- and chromophore-dependent phosphorylation of both Pr and Pfr was found but initially the signals could not be clearly assigned to either auto- or trans-phosphorylation (Blumenstein et al., 2005; Brandt et al., 2008). Recently, Brandt et al. found a strong red light dependent auto-phosphorylation activity of FphA, thus the Pfr form appears to be an active histidine kinase. However, auto-phosphorylation of the Pr form could also be observed and depends on a functional RRD of the dimerization partner. This suggests a functional RRD necessary for tuning the kinase activity in both spectral forms. Subsequently a phosphotransfer from the HKD to the RRD of the dimerization partner appears and this process is independent of the bound chromophore in the phosphoaccepting RRD. Thus trans-phosphorylation also occurs within heterodimerization of holo- and apo-FphA variants (Brandt et al., 2008). Very likely a phosphotransfer initiated from the sensor histidine kinase FphA plays a role in further light signaling cascades.

Therefore, the identification of FphA interacting proteins appeared to be crucial to better understand the signaling process. Indeed it has been discovered that FphA forms a light-regulator complex together with proteins similar to the $N$. crassa white collar proteins, named LreA/LreB and in addition with VeA (Purschwitz et al., 2008) (Fig. 5B). FphA appeared to interact directly with VeA and LreB. The interactions were mapped to the C-terminal output motif of FphA and additionally VeA was found to be a highly phosphorylated protein (Purschwitz et al., 2009). But so far no downstream phosphotransfer initiated by FphA was shown. Interestingly, the interactions occurred in the nucleus although the phytochrome protein is mainly found in the cytoplasm. Similar to the situation in plants, a nuclear shuttle should occur at some point. The fact that under all conditions large amounts of the protein were found in the cytoplasm could be due to the moderate overexpression of the protein, but it could also indicate a cytoplasmic function besides the nuclear function. However, a light-dependent nuclear shuttle of VeA has been reported (Bayram et al., 2008b; Stinnett et al., 2007).

Further evidence for the $A$. nidulans phytochrome to be involved in a phosphorelay system came from the observation of a retrograde phosphotransfer from the phosphotransmitter YpdA to the conserved aspartate in the RRD of FphA (Azuma et al., 2007). Yet, it is unclear, how the interacting proteins influence the activity of the sensor histidine kinase FphA.

\section{Blue-light sensing - the White Collar System}

Blue light responses have been observed in many different fungi from zygomycetes to ascomycetes and basidiomycetes. The response has been best studied in N. crassa, which developed into a model system for light regulation in fungi. Blue-light responses in $N$. crassa include the induction of sporulation and protoperithecia development (Degli-Innocenti et al., 1984), positive phototropism of perithecial beaks (Harding and Melles, 1983), the induction of the synthesis of carotenoids (Harding and Turner, 1981), and the regulation of the circadian clock (Sargent and Briggs, 1967). All the different responses require the $w c-1$ and $w c-2$ genes (Ballario and Macino, 1997; Linden and Macino, 1997). Both N. crassa WC proteins contain a zinc-finger motif for DNA binding, light, oxygene and 


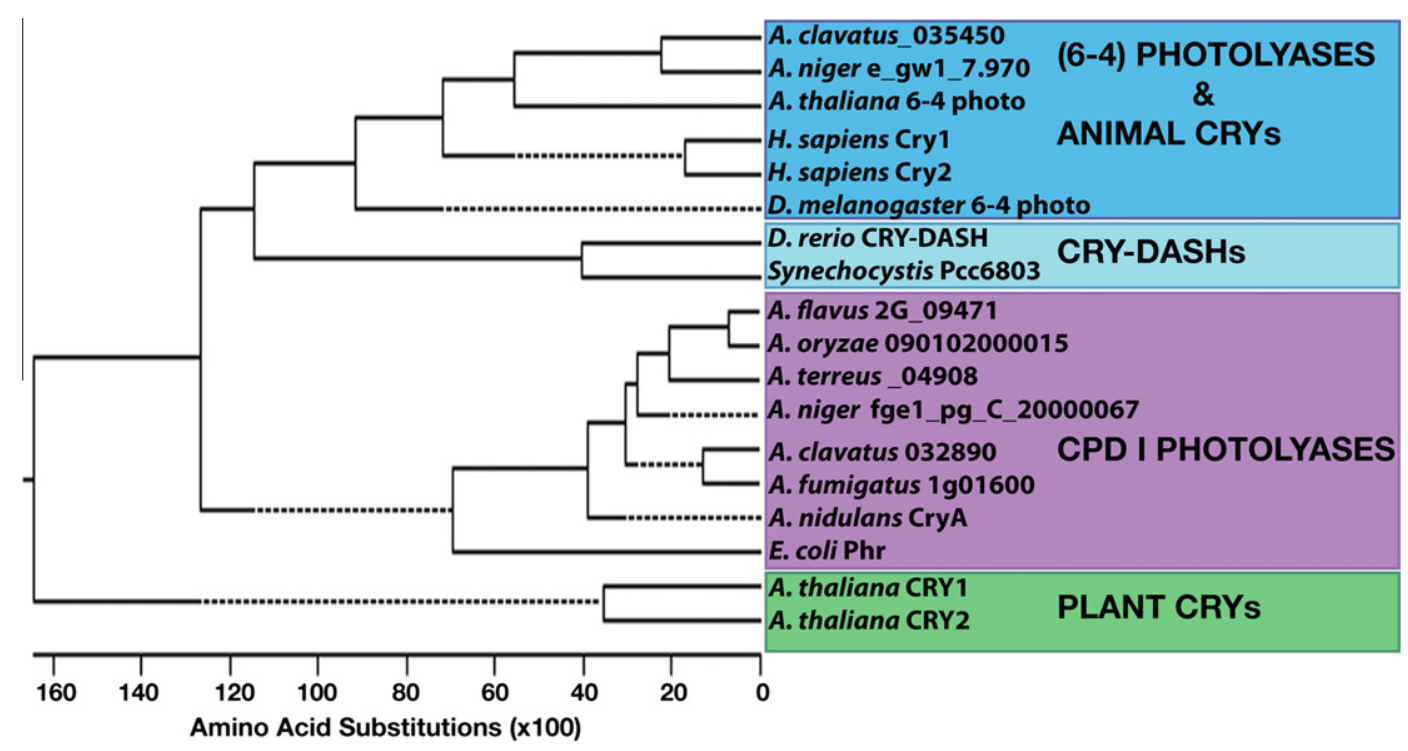

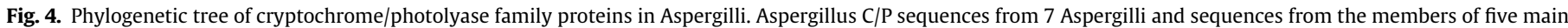

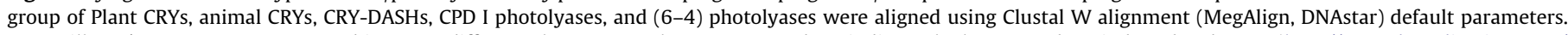

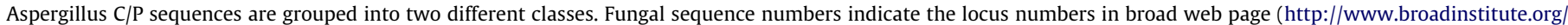
science/data\#).

voltage (LOV) domain for flavin binding, and PAS domains for protein-protein interactions (Ballario et al., 1996; Crosthwaite et al., 1997). WC-1 and WC-2 interact and form the white-collar complex (WCC). This complex, upon light exposure, binds transiently to the promoters of light-inducible genes, presumably to activate their transcription (Belden et al., 2007; Froehlich et al., 2002; He et al., 2002). One of the most interesting open questions was of course the one concerning the photoreceptor. This question was solved simultaneously in two laboratories. Most surprisingly, WC-1, the transcription factors itself, harbors a flavine (FAD) as light-sensing chromophore (Froehlich et al., 2002; He et al., 2002). WC-1 is therefore related to phototropins of plants with which it also shares other protein domains.

The WC-light sensing system has been studied in a number of fungi, such as the ascomycetes Trichoderma (Gressel and Galun, 1967), Magnaporthe grisea (now called M. oryzae) (Lee et al., 2006) and Bipolaris oryzae (Kihara et al., 2004). The discovery of these proteins in ascomycetes, basidiomycetes like Cryptococcus neoformans, and in zygomycetes like Phycomyces blakesleeanus and their corresponding functions as light sensors clearly suggests that the WCC arose early in fungal evolution as a photoreceptor and a transcription factor to regulate fungal photoresponses (Corrochano, 2007; Herrera-Estrella and Horwitz, 2007; Purschwitz et al., 2006, 2008; Sanz et al., 2009). Blue-light sensing has recently also been found in $A$. nidulans where some additional and surprising aspects of the WCC have been discovered (Purschwitz et al.,
2008). Photobiological experiments revealed that the light effect on the morphogenetic pathways integrates blue and red light. Full stimulation of conidia production was only achieved with a combination of red and blue light (Fig. 2A). In contrast, blue light was as effective as white light to repress the production of the mycotoxin sterigmatocystin (Figs. 1 and 2A). The corresponding WC orthologues have been characterized and named LreA (WC-1) and LreB (WC-2). Both proteins have essentially the same domains as described previously for $N$. crassa (Fig. 3). Asexual conidiospore production was slightly increased in $\triangle$ lreA and $\Delta$ lreB strains, independent of the presence or absence of light (Fig. 2B). This suggests a repressing function of LreA and LreB for the asexual cycle. Interestingly, double mutation of lreA or lreB with $f p h A$ or the triple mutation caused a drastic decrease in the number of conidiospores. Thus a synergistic effect occurs between blue- and red-light photoreceptors as it was observed already at the phenotypic level of the light response (see red + blue in Fig. 2A). On the other hand they act as positive factors for the sexual cycle. This function is also required in the dark. The complete loss of cleistothecium formation in the $\Delta$ lreA or $\Delta$ lreB strains in light was most surprisingly, largely suppressed by deletion of $f p h A$. Double and triple mutants of lre $A$, lre $B$, and $f p h A$ incubated in the light produced the same number of cleistothecia as in the dark (Fig. 2B). Most interestingly, LreB interacted not only with LreA, but also with the phytochrome FphA, which itself also interacted with the VeA regulatory protein (Fig. 5B). These results suggest a white-collar phytochrome containing light-regulator
A

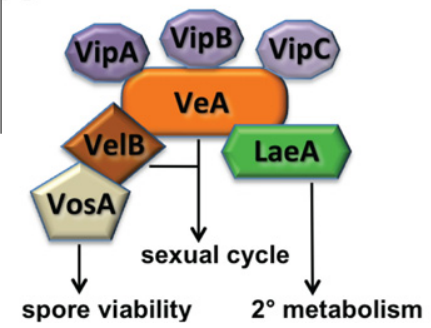

B

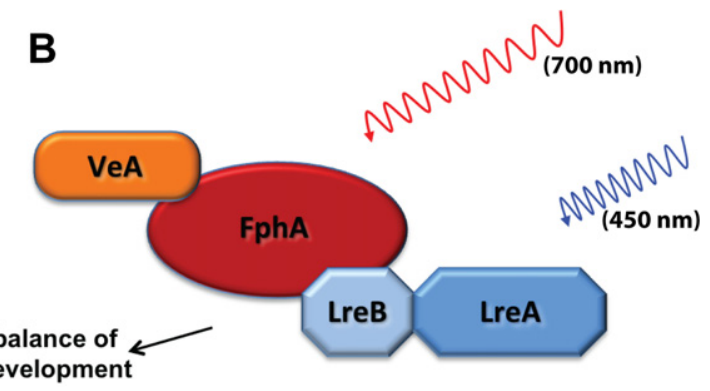

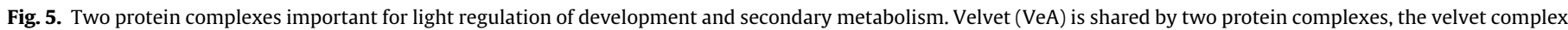

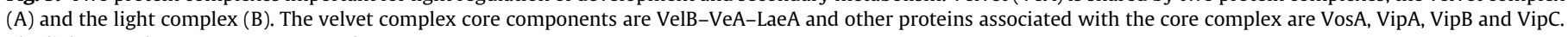
The light complex components are FphA-VeA-LreB-LreA. 
complex. Surprisingly, VeA is also a component of another protein complex with proteins regulating secondary metabolism and development (see below).

\section{Blue and UVA light sensing - the cryptochrome/photolyase CryA}

Cryptochromes are chromoproteins, which are highly conserved from bacteria to human. They act as blue and UVA light receptors that percept light and control entrainment of the circadian rhythyms, regulate development as well as many other responses (Lin and Todo, 2005). There are three divisions of cryptochromes: the plant cryptochromes, the animal cryptochromes, and the Drosophila Arabidopsis, Synechocystis, Human (CRY-DASH) cryptochromes (Brudler et al., 2003).

These proteins for light perception have presumably evolved from DNA photolyase proteins which are part of the direct light response and repair UV lesions. The separation of cryptochrome and DNA photolyase genes is very likely to be ancient. They are already separated in prokaryotes where genes for photolyases as well as cryptochromes are present in numerous genomes. Cryptochromes and photolyases carry a flavin-adenine-dinucleotide (FAD) and in most cases a 5,10-methenyltetrahydrofolate (MTHF) molecule as chromophore (Muller and Carell, 2009). The photolyases are activated by light to repair the major consequence of $U_{V C} 260-210 \mathrm{~nm}$ irradiation, which are cyclobutane pyrimidine dimers (CPD) and in most cases T-T (thymine) dimers (Sancar, 2003). There are two divisions of photolyases, the class I CPD photolyases and the (6-4) photolyases which have some similarity to animal cryptochromes (Lin and Todo, 2005; Muller and Carell, 2009). Cryptochromes have normally lost the DNA repair activity and regulate instead gene expression either upon blue light illumination or irrespective of light. Only the CRY-DASH type proteins where the exact regulatory role is yet obscure, exhibit a relatively low single strand DNA repair activity (Pokorny et al., 2008; Selby and Sancar, 2006). The genomes of many organisms including prokaryotes, plants, mammals, fish, insects contain more than one gene for CPD photolyase, CRY-DASH, (6-4) photolyase and cryptochrome, respectively.

The knowledge about fungal cryptochrome/photolyases $(C / P)$ has been limited for many years. A couple of fungal photolyase/ cryptochrome from ascomycetes and basidiomycetes have been recently investigated (Berrocal-Tito et al., 2007; Bluhm and Dunkle, 2008; Froehlich et al., 2010; Veluchamy and Rollins, 2008). As in other organisms, most fungi possess more than one gene of the photolyase/cryptochrome family. Fig. 4 represents a phylogenetic tree consisting of the genomes of all Aspergilli including $A$. nidulans, A. fumigatus, A. flavus, A. oryzae, A. niger, Aspergillus clavatus, and Aspergillus terreus. The fungal cryptochrome/photolyase phylogenetic tree was constructed by using E. coli CPD I photolyase Phr, Synechocystis (Cyanobacterium) and Danio rerio (Zebrafish) CRYDASH, Arabidopsis thaliana cryptochromes CRY1-2, Homo sapiens cryptochromes Cry1-2, and A. thaliana and Drosophila melanogaster 6-4 photolyases as standards.

All CPD photolyase type I proteins are grouped together with the $E$. coli photolyase sequence confirming that these proteins are at least related to photolyases and their structure. Most Aspergilli genomes contain only one cryptochrome/photolyase-like gene. A. nidulans, A. fumigatus, A. flavus, $A$. terreus, or $A$. oryzae have lost the CRY or CRY-DASH genes presumably through genomic arrangements during evolution (Galagan et al., 2005; Machida et al., 2005; Nierman et al., 2005). Only A. clavatus and A. niger have a CRY-like protein as well as a classical CPD photolyase (Pel et al., 2007), one of which alingns with 6-4 photolyase/animal CRYs group whereas the other one is grouped into the classical CPD photolyase group (Fig. 4). Experimental data for the different divisions of fungal cryptochrome/photolyases are only present for a small number of fungi.

The $A$. nidulans cryA gene represents the single $\mathrm{C} / \mathrm{P}$ of the Aspergilli and is classified as CPD I photolyase. The analysis of the cryA function resulted in the discovery of the first combined cryptochrome/photolyase which further corroborates the common roots of photolyases and cryptochromes during evolution (Bayram et al., 2008a). Recently a second dual function protein of the $C / P$ family has been discovered outside the fungal kingdom in marine diatomes (Coesel et al., 2009). A. nidulans CryA is a bona fide cryptochrome, because it controls the number of fruiting bodies under $\mathrm{UVA}_{350-370 \mathrm{~nm}}$ as well as blue light $450 \mathrm{~nm}$ conditions. In addition, CryA functions as repressor of development and therefore deletion of cryA in submerged culture triggers production of nursing Hülle cells which are normally generated on the plates under dark conditions in order to provide nutrients to developing/maturating cleistothecia. Hülle cell production timing clearly coincides with the upregulation of regulatory proteins of fruiting body formation. CryA has the second function of a photolyase, because it is capable of repairing UV induced CPD dimers when heterelogously expressed in E. coli and overexpressed in A. nidulans.

This leads to the question why some organisms as the Aspergilli have presumably lost CRY-like genes and only kept photolyase-like genes, whereas most fungal genomes have conserved more than one gene of the photolyase/cryptochrome familiy proteins. One possibility is that the Aspergillus cryA gene encoded a functional photolyase which subsequently has acquired additional regulatory functions which made the cryptophrome genes dispensible. The CryA protein of $A$. nidulans regulates the expression of genes for sexual regulators. The molecular details how this mechanism works and also the interaction partners of CryA are currently unknown. It is also unknown whether the regulatory function of CryA requires its binding to DNA to regulate, e.g. promoter activity of target genes.

If regulatory roles of $\mathrm{C} / \mathrm{P}$ genes or other photoreceptors are taken over by other proteins, gene loss seems to be possible. If organisms have to respond to white light white collar proteins which respond to blue light or the FphA phytochrome which responds to red light can even take over some cryptochrome functions. The Aspergillus LreA/LreB/FphA complex might carry already redundant funtions which might reflect overlap between functions of these photoreceptors. This is supported by the finding that the phenotype of lreA or lreB deletions is only visible in the fphA deletion background. Therefore FphA can mask the effect of a single lreA or Ire $B$ deletion, because there is redundancy between LreA or LreB or even FphA might have some overlapping functions. It is currently unknown whether CryA can interact with components of the FphA system or whether we have two separate systems. It is also currently unknown how one molecule can exhibit photolyase and regulatory function and whether the two functions can be separated. Future experiments will have to reveal the enigmatic nature of dual function cryptochrome/photolyase proteins of Aspergilli.

\section{The velvet complex: branchpoint of light signaling in coordinated control of development and secondary metabolism}

Since its first description by Kaefer (1965) the A. nidulans velvet gene and its gene product VeA and the veA1 mutation which is commonly used in laboratory strains remained a mystery. In 2002, Kim and co-workers (Kim et al., 2002) demonstrated that the veA1 allele comprises a base substitution in the start codon of $v e A$ gene, which resulted in a change from ATG to ATT. The $\mathrm{N}$-terminus of the VeA1 protein is truncated, because translation starts at the 37th codon which is a methionine. This result of Kim and co-workers implied the importance of the $\mathrm{N}$-terminus of 
VeA for the development since strains bearing the truncated VeA1 version are unaffected by illumination and do not manifest lightdark distinction in terms of cleistothecia production. Deletion of the veA gene results in an acleistothecial phenotype even under conditions that promote sexual development, whereas overexpression triggers the formation of sexual cell types under unappropriate conditions. Velvet proteins are highly conserved through the filamentous fungi as extensively reviewed recently by Calvo (2008, and references cited there).

In the meantime VeA of $A$. nidulans has been found to interact to the phytochrome-white collar light-regulator complex (see above) and VeA is part of the velvet complex VelB/VeA/LaeA. This complex coordinates the light signal with fungal development and secondary metabolism (Bayram et al., 2008b) (Fig. 5A). A. nidulans produces various secondary metabolites which might be required for the relationship to the other inhabitants of the soil to communicate or to defend the fungus against eukaryotic or prokaryotic competitors (Keller et al., 2005). The A. nidulans product sterigmatocystin (ST) is a mycotoxin which is a precursor of aflatoxin produced by $A$. flavus (Bhatnagar et al., 2003; Brase et al., 2009). A. nidulans is also able to produce the antibiotic penicillin (Brakhage et al., 2004). The involvement of VeA in secondary metabolism control was originally shown for sterigmatocystin biosynthesis where VeA is required (Kato et al., 2003). Kato and co-workers also found that penicillin production is affected by VeA, but the regulatory mechanism has not yet been solved. The ipnA gene encoding isopenicillin synthetase is repressed by VeA whereas acvA encoding another enzyme of the same pathway can be as well activated as repressed by VeA (Kato et al., 2003; Sprote and Brakhage, 2007).

A. nidulans VeA localizes in the nucleus in dark but is predominantly retained in the cytoplasm in light. VeA interacts to the a-importin KapA which presumably provides nuclear import (Stinnett et al., 2007). The truncation of 36 amino acids of the $\mathrm{N}$-terminally shortened VeA1 results in a predominant cytoplasmic fraction which is not altered by illumination. The bipartite nuclear localization signal (NLS) is located in the N-terminus of the protein (Fig. 3) and is disrupted in VeA1. VeA also interacts to the phytochrome FphA which is connected to the white collar proteins LreB and LreA (Purschwitz et al., 2008). This suggests that the light signal is transmitted to the red and blue-light sensing components which might control VeA activity through direct protein-protein interaction. The blue and UV light sensing system component CryA functions by another mechanism. It does not interact with VeA, but represses veA mRNA expression and therefore VeA protein levels within the fungal cell (Bayram et al., 2008a).

The velvet complex consisting of VelB/VeA/LaeA was discovered by biochemical Tandem Affinity Purification (TAP) using a tapped VeA as a bait (Bayram et al., 2008b) (Fig. 5A). A stable trimeric protein complex could only be enriched from dark grown cultures. In the light significantly less VeA was present in the nucleus and the velvet complex was disrupted because the bridging factor is missing. VelB, a novel velvet-like protein, is bridged by VeA to the master regulator of secondary metabolism, a putative histone methyltransferase LaeA (Bok and Keller, 2004). LaeA is required for the expression of many secondary metabolite gene clusters including subtelomeric ST biosynthesis gene cluster. LaeA has been proposed to control methylation of histones like H3K9 (Histone 3, Lysine 9) (Keller et al., 2005) (Bok et al., 2009).

VelB and VeA are members of the family of velvet-like proteins, which also include VelC and VosA, and which is common in almost all ascomycetous fungi ( $\mathrm{Ni}$ and $\mathrm{Yu}, 2007$ ). Components of the velvet complex are also found in opportunistic human pathogens like the dimorphic fungus Histoplasma capsulatum. Ryp2 (VelB homo$\log$ ) and Ryp3 (VosA homolog) proteins of $H$. capsulatum are crucial for the transition from filamentous phase to the yeast phase (Webster and Sil, 2008).
Deletion of the velB gene of $A$. nidulans results in a similar phenotype as a $\triangle v e A$ mutation: loss of sexual fruiting bodies and impaired secondary metabolism represented by secretion of a red-brownish pigment into the agar plate. velB mutants are unable to produce the fungal mycotoxin ST as also observed for veA and laeA mutants.

Sterigmatocystin production levels of veA1 strains are similar to the $v e A$ wild type strain, presumably because there is still a functional C-terminal interaction between VeA1 and LaeA (Fig. 5A). However, the interaction between VeA and VelB is seriously perturbed in the veA1 background since the interaction of VeA and VelB requires the $\mathrm{N}$-terminus of $\mathrm{VeA}$ which is missing in the VeA1 protein. This results in an impairment of development presumably due to weakened/perturbed interaction between VeA and VelB.

VelB might be part of a second complex, because it also interacts to VosA, a protein which is required for viable spores. Deletion of the vosA gene in $A$. nidulans triggers early loss of cytoplasm in asexual and sexual spores, which is brought about by the lack of trehalose. Therefore the tolerance of conidia to heat and oxidative stress is reduced. Apparently, there are more proteins interacting with the velvet complex including Vip proteins VipA, VipB, and VipC (Calvo, 2008), although the function of these interactions remain to be determined.

The relationship of the different complexes where VeA seems to be part of is currently unsolved (Fig. 5). We assume that the trimeric core complex containing VelB-VeA-LaeA is rather stable when developmental competence is established. This is supported by the enrichment of this complex at this developmental time point by TAP purification. The purification revealed no other protein and X-ray crystallography will be required to solve whether this is a stoichiometric complex. The second VeA complex, which is the phytochrome-white collar containing light-regulator complex comprising VeA-FphA-LreA-LreB is either formed under different physiological or developmental conditions than the VelB-VeA-LaeA complex or could be also a more transient complex within the light signal pathway.

\section{Outlook}

The molecular analysis of the photobiology of $A$. nidulans revealed several fascinating novel aspects, among which are the cross-talk between the red- and the blue-light sensing system and the interplay between the two morphogenetic pathways and the secondary metabolism. The key for the understanding of these phenomena lies probably in the understanding of two recently discovered important protein complexes, which both share the fungal-specific regulator VeA. Therefore one of the main goals for future research will be the analysis of the phytochrome white-collar light-regulator complex and of the VelB-VeA-LaeA complex and the interplay between these complexes. Some major future questions concern the exact composition of the complexes in different tissues or at different times, the assembly of the proteins, and the molecular events within the complexes following light exposure. In order to understand the biological responses following illumination, it is crucial to identify light-regulated genes and analyse the exact mechanisms of induction or repression. The large number of light-regulated genes in N. crassa and other fungi highlights the importance of light regulation and suggests that most morphogenetic or metabolic pathways are influenced by light, although perhaps to different extents. This may have important consequences for the production of secondary and other metabolites in biotechnology but also for the control of pathogenic fungi. After some decades where light as signal for fungal life has been neglected, we currently see a revival of this research which should lead to interesting novel discoveries. 


\section{Acknowledgments}

The work of the Göttingen group has been funded by grants from DFG, the Volkswagen-Stiftung, and the Fonds der Chemischen Industrie. The work of the Karlsruhe group has been supported by the DFG, the Fonds der Chemischen Industrie, the Center for Functional Nanostructures, and the Baden-Württemberg Stiftung. J.R. is a fellow of the Spanish Science Ministry (postdoctoral MEC fellowship).

\section{References}

Adams, T.H., Deising, H., Timberlake, W.E., 1990. brlA requires both zinc fingers to induce development. Mol. Cell Biol. 10, 1815-1817.

Adams, T.H., Wieser, J.K., Yu, J.H., 1998. Asexual sporulation in Aspergillus nidulans Microbiol. Mol. Biol. Rev. 62, 35-54.

Andrianopoulos, A., Timberlake, W.E., 1994. The Aspergillus nidulans abaA gene encodes a transcriptional activator that acts as a genetic switch to control development. Mol. Cell. Biol. 14, 2503-2515.

Azuma, N., Kanamaru, K., Matsushika, A., Yamashino, T., Mizuno, T., Kato, M., Kobayashi, T., 2007. In vitro analysis of His-Asp phosphorelays in Aspergillu nidulans: the first direct biochemical evidence for the existence of His-Asp phosphotransfer systems in filamentous fungi. Biosci. Biotechnol. Biochem. 71, 2493-2502.

Ballario, P., Macino, G., 1997. White collar proteins: PASsing the light signal in Neurospora crassa. Trends Microbiol. 5, 458-462.

Ballario, P., Vittorioso, P., Magrelli, A., Talora, C., Cabibbo, A., Macino, G., 1996. White collar-1, a central regulator of blue light responses in Neurospora, is a zinc finger protein. EMBO J. 15, 1650-1657.

Bayram, O., Biesemann, C., Krappmann, S., Galland, P., Braus, G.H., 2008a. More than a repair enzyme: Aspergillus nidulans photolyase-like CryA is a regulator of sexual development. Mol. Biol. Cell. 19, 3254-3262.

Bayram, O., Krappmann, S., Ni, M., Bok, J.W., Helmstaedt, K., Valerius, O., BrausStromeyer, S., Kwon, N.J., Keller, N.P., Yu, J.H., Braus, G.H., 2008b. VelB/VeA/LaeA complex coordinates light signal with fungal development and secondary metabolism. Science 320, 1504-1506.

Belden, W.J., Loros, J.J., Dunlap, J.C., 2007. Execution of the circadian negative feedback loop in Neurospora requires the ATP-dependent chromatinremodeling enzyme CLOCKSWITCH. Mol. Cell. 25, 587-600.

Berrocal-Tito, G.M., Esquivel-Naranjo, E.U., Horwitz, B.A., Herrera-Estrella, A., 2007. Trichoderma atroviride PHR1, a fungal photolyase responsible for DNA repair, autoregulates its own photoinduction. Eukaryot. Cell 6, 1682-1692.

Bhatnagar, D., Ehrlich, K.C., Cleveland, T.E., 2003. Molecular genetic analysis and regulation of aflatoxin biosynthesis. Appl. Microbiol. Biotechnol. 61, 83-93.

Bluhm, B.H., Dunkle, L.D., 2008. PHL1 of Cercospora zeae-maydis encodes a member of the photolyase/cryptochrome family involved in UV protection and fungal development. Fungal Genet. Biol. 45, 1364-1372.

Blumenstein, A., Vienken, K., Tasler, R., Purschwitz, J., Veith, D., Frankenberg-Dinkel, N., Fischer, R., 2005. The Aspergillus nidulans phytochrome FphA represse sexual development in red light. Curr. Biol. 15, 1833-1838.

Bok, J.W., Chiang, Y.M., Szewczyk, E., Reyes-Dominguez, Y., Davidson, A.D., Sanchez, J.F., Lo, H.C., Watanabe, K., Strauss, J., Oakley, B.R., Wang, C.C., Keller, N.P., 2009 Chromatin-level regulation of biosynthetic gene clusters. Nat. Chem. Biol. 5 462-464.

Bok, J.W., Keller, N.P., 2004. LaeA, a regulator of secondary metabolism in Aspergillus spp. Eukaryot. Cell 3, 527-535.

Brakhage, A.A., Sprote, P., Al-Abdallah, O., Gehrke, A., Plattner, H., Tuncher, A., 2004 Regulation of penicillin biosynthesis in filamentous fungi. Adv. Biochem. Eng. Biotechnol. 88, 45-90.

Brandt, S., von Stetten, D., Gunther, M., Hildebrandt, P., Frankenberg-Dinkel, N. 2008. The fungal phytochrome FphA from Aspergillus nidulans. J. Biol. Chem. 283, 34605-34614.

Brase, S., Encinas, A., Keck, J., Nising, C.F., 2009. Chemistry and biology of mycotoxins and related fungal metabolites. Chem. Rev. 109, 3903-3990.

Braus, G.H., Krappmann, S., Eckert, S.E., 2002. Sexual development in ascomycetes Fruit body formation of Aspergillus nidulans. In: Osiewacz, H.D. (Ed.), Molecular Biology of Fungal Development. Marcel Dekker, Inc., New York, Basel, pp. 215244.

Brudler, R., Hitomi, K., Daiyasu, H., Toh, H., Kucho, K., Ishiura, M., Kanehisa, M., Roberts, V.A., Todo, T., Tainer, J.A., Getzoff, E.D., 2003. Identification of a new cryptochrome class. Structure, function, and evolution. Mol. Cell. 11, 59-67.

Busch, S., Braus, G.H., 2007. How to build a fungal fruit body: from uniform cells to specialized tissue. Mol. Microbiol. 64, 873-876.

Calvo, A.M., 2008. The VeA regulatory system and its role in morphological and chemical development in fungi. Fungal Genet. Biol. 45, 1053-1061.

Calvo, A.M., Wilson, R.A., Bok, J.W., Keller, N.P., 2002. Relationship between secondary metabolism and fungal development. Microbiol. Mol. Biol. Rev. 66, 447-459 (table of content).

Clutterbuck, A.J., 1969. A mutational analysis of conidial development in Aspergillus nidulans. Genetics 63, 317-327.

Coesel, S., Mangogna, M., Ishikawa, T., Heijde, M., Rogato, A., Finazzi, G., Todo, T., Bowler, C., Falciatore, A., 2009. Diatom PtCPF1 is a new cryptochrome/ photolyase family member with DNA repair and transcription regulation activity. EMBO Rep. 10, 655-661.

Corrochano, L.M., 2007. Fungal photoreceptors: sensory molecules for fungal development and behaviour. Photochem. Photobiol. Sci. 6, 725-736.

Crosthwaite, S.K., Dunlap, J.C., Loros, J.J., 1997. Neurospora wc-1 and wc-2: Transcription, photoresponses, and the origins of circadian rhythmicity. Science 276, 763-769.

Davis, S.J., Vener, A.V., Vierstra, R.D., 1999. Bacteriophytochromes: phytochromelike photoreceptors from nonphotosynthetic eubacteria. Science 286, 25172520 .

Degli-Innocenti, F., Chambers, J.A., Russo, V.E., 1984. Conidia induce the formation of protoperithecia in Neurospora crassa: further characterization of white collar mutants. J. Bacteriol. 159, 808-810.

Draht, O.W., Busch, S., Hofmann, K., Braus-Stromeyer, S., Helmstaedt, K., Goldman, G.H., Braus, G.H., 2007. Amino acid supply of Aspergillus. In: Goldman, Osmani (Eds.), The Aspergilli: Genomics, Medical, Aspects Biotechnology and Research Methods. CRC Press Taylor \& Francis Group, Boca Raton FL (USA), pp. 143-175.

Fischer, R., Kües, U., 2006. A sexual sporulation in mycelial fungi. In: Kües, U., Fischer, R. (Eds.), The Mycota, Growth differentiation and sexuality. Springer, Heidelberg, pp. 263-292.

Froehlich, A.C., Chen, C.H., Belden, W.J., Madeti, C., Roenneberg, T., Merrow, M., Loros, J.J., Dunlap, J.C., 2010. Genetic and molecular characterization of a cryptochrome from the filamentous fungus Neurospora crassa. Eukaryot. Cell 9, 738-750.

Froehlich, A.C., Liu, Y., Loros, J.J., Dunlap, J.C., 2002. White Collar-1, a circadian blue light photoreceptor, binding to the frequency promoter. Science 297, 815-819.

Froehlich, A.C., Noh, B., Vierstra, R.D., Loros, J., Dunlap, J.C., 2005. Genetic and molecular analysis of phytochromes from the filamentous fungus Neurospora crassa. Eukaryot. Cell 4, 2140-2152.

Galagan, J.E., Calvo, S.E., Cuomo, C., Ma, L.J., Wortman, J.R., Batzoglou, S., Lee, S.I., Basturkmen, M., Spevak, C.C., Clutterbuck, J., Kapitonov, V., Jurka, J., Scazzocchio, C., Farman, M., Butler, J., Purcell, S., Harris, S., Braus, G.H., Draht, O., Busch, S., D'Enfert, C., Bouchier, C., Goldman, G.H., Bell-Pedersen, D., Griffiths-Jones, S. Doonan, J.H., Yu, J., Vienken, K., Pain, A., Freitag, M., Selker, E.U., Archer, D.B., Penalva, M.A., Oakley, B.R., Momany, M., Tanaka, T., Kumagai, T., Asai, K., Machida, M., Nierman, W.C., Denning, D.W., Caddick, M., Hynes, M., Paoletti, M., Fischer, R., Miller, B., Dyer, P., Sachs, M.S., Osmani, S.A., Birren, B.W., Eckert, S.E., Krappmann, S., 2005. Sequencing of Aspergillus nidulans and comparative analysis with $A$. fumigatus and $A$. oryzae. Nature 438, 1105-1115.

Gressel, J., Galun, E., 1967. Morphogenesis in Trichoderma: photoinduction and RNA. Dev. Biol. 15, 575-598.

Gugnani, H.C., 2003. Ecology and taxonomy of pathogenic aspergilli. Front. Biosci. 8, s346-357.

Han, K.H., Han, K.Y., Yu, J.H., Chae, K.S., Jahng, K.Y., Han, D.M., 2001. The nsdD gene encodes a putative GATA-type transcription factor necessary for sexual development of Aspergillus nidulans. Mol. Microbiol. 41, 299-309.

Harding, R.W., Melles, S., 1983. Genetic analysis of phototropism of Neurospora crassa perithecial beaks using white collar and albino mutants. Plant Physiol. 72, 996-1000.

Harding, R.W., Turner, R.V., 1981. Photoregulation of the carotenoid biosynthetic pathway in albino and white collar mutants of Neurospora crassa. Plant Physiol. 68, 745-749.

He, Q., Cheng, P., Yang, Y., Wang, L., Gardner, K.H., Liu, Y., 2002. White collar-1, a DNA binding transcription factor and a light sensor. Science 297, 840-843.

Hedayati, M.T., Pasqualotto, A.C., Warn, P.A., Bowyer, P., Denning, D.W., 2007. Aspergillus flavus: human pathogen, allergen and mycotoxin producer. Microbiology 153, 1677-1692.

Herrera-Estrella, A., Horwitz, B.A., 2007. Looking through the eyes of fungi: molecular genetics of photoreception. Mol. Microbiol. 64, 5-15.

Kaefer, E., 1965. Origins of translocations in Aspergillus nidulans. Genetics 52, 217 232.

Kaneko, T., Sato, S., Kotani, H., Tanaka, A., Asamizu, E., Nakamura, Y., Miyajima, N., Hirosawa, M., Sugiura, M., Sasamoto, S., Kimura, T., Hosouchi, T., Matsuno, A., Muraki, A., Nakazaki, N., Naruo, K., Okumura, S., Shimpo, S., Takeuchi, C., Wada, T., Watanabe, A., Yamada, M., Yasuda, M., Tabata, S., 1996. Sequence analysis of the genome of the unicellular cyanobacterium Synechocystis sp. strain PCC6803. II. Sequence determination of the entire genome and assignment of potential protein-coding regions. DNA Res. 3, 109-136.

Karniol, B., Wagner, J.R., Walker, J.M., Vierstra, R.D., 2005. Phylogenetic analysis of the phytochrome superfamily reveals distinct microbial subfamilies of photoreceptors. Biochem. J. 392, 103-116.

Kato, N., Brooks, W., Calvo, A.M., 2003. The expression of sterigmatocystin and penicillin genes in Aspergillus nidulans is controlled by $v e A$, a gene required for sexual development. Eukaryot. Cell 2, 1178-1186.

Kehoe, D.M., Grossman, A.R., 1996. Similarity of a chromatic adaptation sensor to phytochrome and ethylene receptors. Science 273, 1409-1412.

Keller, N.P., Turner, G., Bennett, J.W., 2005. Fungal secondary metabolism - from biochemistry to genomics. Nat. Rev. Microbiol. 3, 937-947.

Kihara, J., Moriwaki, A., Ueno, M., Tokunaga, T., Arase, S., Honda, Y., 2004. Cloning functional analysis and expression of a scytalone dehydratase gene (SCD1) involved in melanin biosynthesis of the phytopathogenic fungus Bipolaris oryzae. Curr. Genet. 45, 197-204.

Kim, D., Yukl, E.T., Moenne-Loccoz, P., Montellano, P.R., 2006. Fungal heme oxygenases: functional expression and characterization of Hmx1 from Saccharomyces cerevisiae and CaHmx1 from Candida albicans. Biochemistry 45 , $14772-14780$ 
Kim, H., Han, K., Kim, K., Han, D., Jahng, K., Chae, K., 2002. The veA gene activates sexual development in Aspergillus nidulans. Fungal Genet. Biol. 37, 72-80.

Lamparter, T., Mittmann, F., Gartner, W., Borner, T., Hartmann, E., Hughes, J., 1997. Characterization of recombinant phytochrome from the cyanobacterium Synechocystis. Proc. Natl. Acad. Sci. USA 94, 11792-11797.

Lee, B.N., Adams, T.H., 1994. Overexpression of $f l b A$, an early regulator of Aspergillus asexual sporulation, leads to activation of $\operatorname{brl} A$ and premature initiation of development. Mol. Microbiol. 14, 323-334.

Lee, K., Singh, P., Chung, W.C., Ash, J., Kim, T.S., Hang, L., Park, S., 2006. Light regulation of asexual development in the rice blast fungus, Magnaporthe oryzae. FGB 43, 694-706.

Lewis, Z.A., Correa, A., Schwerdtfeger, C., Link, K.L, Xie, X, Gomer, R.H., Thomas, T. Ebbole, D.J., Bell-Pedersen, D., 2002. Overexpression of White Collar-1 (WC-1) activates circadian clock-associated genes, but is not sufficient to induce most light-regulated gene expression in Neurospora crassa. Mol. Microbiol. 45, 917931.

Lin, C., Todo, T., 2005. The cryptochromes. Genome Biol. 6, 220.

Linden, H., Macino, G., 1997. White collar 2, a partner in blue-light signal transduction, controlling expression of light-regulated genes in Neurospora crassa. EMBO J. 16, 98-109.

Lukens, R.J., 1965. Reversal by red light of blue light inhibition of sporulation in Alternaria solani. Phytophathology 55, 1032.

Mooney, J.L., Yager, L.N., 1990. Light is required for conidiation in Aspergillus nidulans. Genes Dev. 4, 1473-1482.

Muller, M., Carell, T., 2009. Structural biology of DNA photolyases and cryptochromes. Curr. Opin. Struct. Biol. 19, 277-285.

Ni, M., Yu, J.H., 2007. A novel regulator couples sporogenesis and trehalose biogenesis in Aspergillus nidulans. PLoS One 2, e970.

Pel, H.J. et al., 2007. Genome sequencing and analysis of the versatile cell factory Aspergillus niger CBS 513. 88. Nat. Biotechnol. 25, 221-231.

Pöggeler, S., Nowrousian, M., Kück, U., 2006. Fruiting-body development in ascomycetes. In: Fischer, K. (Ed.), The Mycota I Growth, Differentiation and Sexuality. Springer-Verlag, Heidelberg, pp. 325-355.

Purschwitz, J., Muller, S., Fischer, R., 2009. Mapping the interaction sites of Aspergillus nidulans phytochrome FphA with the global regulator VeA and the White Collar protein LreB. Mol. Genet. Genomics. 281, 35-42.

Purschwitz, J., Muller, S., Kastner, C., Fischer, R., 2006. Seeing the rainbow: light sensing in fungi. Curr. Opin. Microbiol. 9, 566-571.

Purschwitz, J., Muller, S., Kastner, C., Schoser, M., Haas, H., Espeso, E.A., Atoui, A., Calvo, A.M., Fischer, R., 2008. Functional and physical interaction of blue- and red-light sensors in Aspergillus nidulans. Curr. Biol. 18, 255-259.

Quail, P.H., 2002. Phytochrome photosensory signalling networks. Nat. Rev. Mol. Cell Biol. 3, 85-93.
Rockwell, N.C., Su, Y.S., Lagarias, J.C., 2006. Phytochrome structure and signaling mechanisms. Annu. Rev. Plant Biol. 57, 837-858.

Rosales-Saavedra, T., Esquivel-Naranjo, E.U., Casas-Flores, S., Martínez-Hernández P., Olmedo-Monfil, V., Herrera-Estrella, A., 2006. Cross talk between a fungal blue-light perception system and the cyclic AMP signaling pathway. Eukaryot. Cell 5, 499-506.

Sakamoto, K., Nagatani, A., 1996. Nuclear localization activity of phytochrome B. Plant J. 10, 859-868.

Samson, R.A., 1992. Current taxonomic schemes of the genus Aspergillus and its teleomorphs. Biotechnol. 23, 355-390.

Sancar, A., 2003. Structure and function of DNA photolyase and cryptochrome blue light-photoreceptors. Chem. Rev. 103, 2203-2237.

Sanz, C., Rodriguez-Romero, J., Idnurm, A., Christie, J.M., Heitman, J., Corrochano L.M., Eslava, A.P., 2009. Phycomyces MADB interacts with MADA to form the primary photoreceptor complex for fungal phototropism. Proc. Natl. Acad. Sci. USA 106, 7095-7100.

Sargent, M.L., Briggs, W.R., 1967. The effects of light on a circadian rhythm of conidiation in Neurospora. Plant Physiol. 42, 1504-1510.

Sprote, P., Brakhage, A.A., 2007. The light-dependent regulator velvet A of Aspergillus nidulans acts as a repressor of the penicillin biosynthesis. Arch. Microbiol. 188 69-79.

Stinnett, S.M., Espeso, E.A., Cobeno, L., Araujo-Bazan, L., Calvo, A.M., 2007. Aspergillus nidulans VeA subcellular localization is dependent on the importin alpha carrie and on light. Mol. Microbiol. 63, 242-255.

Tan, K.K., 1974. Red-far-red reversible photoreaction in the recovery from bluelight inhibition of sporulation in Botrytis cinerea. J. Gen. Microbiol. 8a, 201202.

Timberlake, W.E., 1987. Molecular genetic analysis of development in Aspergillus nidulans. In: Loomis, W.F. (Ed.), Genetic Regulation of Development. Alan R. Liss, Inc., New York, pp. 63-82.

Todd, R.B., Hynes, M.J., Andrianopoulos, A., 2006. The Aspergillus nidulans rcoA gene is required for veA-dependent sexual development. Genetics 174, 1685-1688.

Veluchamy, S., Rollins, J.A., 2008. A CRY-DASH-type photolyase/cryptochrome from Sclerotinia sclerotiorum mediates minor UV-A-specific effects on development. Fungal Genet. Biol. 45, 1265-1276.

Vienken, K., Fischer, R., 2006. The Zn(II)2Cys6 putative transcription factor NosA controls fruiting body formation in Aspergillus nidulans. Mol. Microbiol. 61, 544554.

Webster, R.H., Sil, A., 2008. Conserved factors Ryp2 and Ryp3 control cell morphology and infectious spore formation in the fungal pathogen Histoplasma capsulatum. Proc. Natl. Acad. Sci. USA 105, 14573-14578.

Yu, J.H., Keller, N., 2005. Regulation of secondary metabolism in filamentous fungi. Annu. Rev. Phytopathol. 43, 437-458. 